\title{
Estrategias de Enseñanza y Creatividad del Docente en el área de Ciencias Sociales de Instituciones Educativas de Media de San Francisco 1
}

\author{
Creative Strategies for Teaching and Teachers in the area of Social \\ Sciences Media Educational Institutions of San Francisco 1
}

\author{
Yajaira Blanquiz ${ }^{*}$ y María Flor Villalobos ${ }^{* *}$
}

\begin{abstract}
RESUMEN
Este estudio tuvo como propósito determinar la relación entre las Estrategias de enseñanza y la Creatividad del docente de áreas de Ciencias Sociales de Instituciones Educativas de Media General del Municipio San Francisco 1, estado Zulia; categorizada por ser un paradigma cuantitativo en un enfoque positivista, fundamento bajo las teorías de Gouveia y Atencio (2012), Díaz y Hernández (2010), Correa (2010), Cormack (2009), De Bono (2009), López (2009), entre otros. La misma se ubica en el paradigma positivista; a su vez, el tipo de investigación fue descriptivo y correlacional; con un diseño no experimental, transversal y de campo. La población estuvo constituida por tres directores, noventa y tres docentes y setenta y cinco estudiantes. Se elaboró un cuestionario con 45 ítems para ambos, el cual fue validado por 7 expertos en el área de gerencia y metodología de la investigación. Para la confiabilidad se utilizó el alfa Cronbach, arrojando como resultado 0,84 para los docentes y 0,81 estudiantes. Se concluyó que para los estudiantes las estrategias empleadas en la escuela son aceptables, mientras que para los docentes dichas estrategias son muy deficientes, estas divergencias indican una sentida necesidad de mejoramiento de las estrategias empleadas por parte del personal docente. Asimismo, se recomendó capacitar al personal docente de las instituciones de media general en cuanto al uso y manejo adecuado, acerca de las estrategias de enseñanza, formando el camino para el logro de aprendizaje significativo. Además, trabajar la escucha activa de los equipos de trabajo que permitan la solución de situaciones conflictivas, mejorar la toma de decisiones, respetando la opinión de los demás.
\end{abstract}

\footnotetext{
* Educadora en el área de Ciencias Sociales mención: geografía; Docente coordinadora en el liceo Eduardo Mathías Lossada, trayectoria 15 años de experiencia académica en todas las áreas de las ciencias sociales. Magister en Gerencia Educativa, Dra. Maracaibo, Venezuela. Correo electrónico: yajairablanquiz_1@ hotmail.com

${ }^{* * *}$ Filosofa con perfeccionamiento docente, Lcda. en lengua y literatura, Magister en Ciencias de la comunicación, Doctora en Ciencias de la Educación, Docente en el área de lenguaje, coordinadora académica del área de lenguaje, 28 años de experiencia académica, experiencia administrativa, Universidad del Zulia, actualmente, editora de libros académicos y correctora de estilo de revistas electrónicas, URBE. Cursando postdoctorado en Ciencias Humanas. Maracaibo, Venezuela. Correo electrónico: maríaflorvillalobos@ hotmail.com
} 
Palabra Clave: Estrategias de Enseñanza, Creatividad, Ciencias Sociales.

\section{ABSTRACT}

This study aimed to establish the relationship between teaching strategies and teacher Creativity areas of Social Sciences Media General Educational Institutions San Francisco 1 Municipality, Zulia state; be categorized for quantitative positivistic paradigm approach, basis on the theories of Cormack (2013), Gouveia and Atencio (2012), Diaz and Hernandez (2010), Correa (2010), Cormack (2009), De Bono (2009) Lopez (2008), among others. It is located in the positivist paradigm; in turn, the type of research was descriptive and correlational; with a non-experimental, cross-sectional design field. The population consisted of three directors, teachers and ninety-three and seventy-five students. A questionnaire with 45 items for both, which was validated by 7 experts in the field of management and research methodology was developed. For reliability Cronbach's alpha was used, yielding results in 0.84 and 0.81 for teachers students. It was concluded that for students the strategies used in the school are acceptable, while for those strategies teachers are very poor, these differences indicate a felt need for improvement of the strategies used by the teachers. It is also recommended to train teachers in general secondary institutions in the use and proper handling, about teaching strategies, forming the way for achieving meaningful learning. In addition, active listening work of teams that allow the solution of conflict situations, improve decision making, respecting the opinions of others.

Keyword: Teaching Strategies, Creativity, Social Sciences .

\section{Problemática}

Es pertinente puntualizar que las estrategias de enseñanza deben ser elaboradas en forma directa, precisa, entendible, utilizando una redacción o un vocabulario adecuado para el estudiante; de igual manera, se hace necesario dejar en claro cuando se enuncien las actividades, contenidos, y los resultados esperados que debe promover el docente pedagógico.

En virtud de todo lo expuesto, Cerda (2005, p. 156), cree que es una necesidad de primer orden educar en creatividad, propiciado espacios académicos divergentes. Desde los cuales poder ejercitar y actualizar tanto a discentes como docentes e investigadores en las técnicas o herramientas creativas, las cuales permiten a los individuos dimensionar sus prácticas profesionales para conseguir afectar su entorno 
Estrategias de Enseñanza y Creatividad del Docente en el área de Ciencias Sociales de Instituciones Educativas de Media de San Francisco 1

favorablemente. Para ello es necesario que el docente sea constructivo, reproductivo, organizado, creativo, expresivo, liberador, innovador, transformador.

Pero es necesario que el Sistema Educativo venezolano incorpore estrategias de enseñanza y desarrolle la creatividad en los docentes, mediante metodologías para promover la personalidad creadora de todos los actores del proceso educativo. Debe incluirse programas para desarrollar, junto con el pensamiento lógico, analítico y crítico, aquellos aspectos que aún hoy son bastante negados en el ministerio del poder popular para la Educación, como lo afectivo, la imaginación, lo sensorial, lo sensible y lo artístico. Es decir, una educación basada en el aprendizaje integral, incluyendo la creatividad y sus estrategias como ejes de principales.

A partir de la implementación de la Reforma Educacional en el país, se han llevado a cabo una serie de programas con la finalidad de entregar herramientas a las instituciones y a profesores para la aplicación o utilización de nuevas estrategias de enseñanza. Pero los cambios, evidenciados en los resultados de las pruebas nacionales, demuestran ser muy lentos. Incluso se ha detectado que los niveles de educación media general, la modificación de las prácticas educacionales es casi nula.

Igualmente, según el Primer Plan Socialista: Proyecto Nacional Simón Bolívar (República Bolivariana de Venezuela, 2007-2013),

"hay que profundizar la universalización de la educación bolivariana, garantizando la permanencia y prosecución en el sistema educativo, mientras se fortalezca e incentive la investigación en el proceso pedagógico, incorporando las nuevas tecnologías de la información y la comunicación, permitiendo el acceso al conocimiento para expandir la educación superior con pertinencia" (p. 9).

Pese a la comprobada insatisfacción de la orientación conductual respecto al aprendizaje, profesores de media general del estado Zulia siguen actuando en sus clases de acuerdo con la metáfora de adquisición de respuestas .El docente parece resistirse a la mentalización profesional acorde con la nueva sociedad y mantiene los mismos métodos o técnicas de enseñanza tradicionales. Se puede observar también que su experiencia no sintoniza con la praxis educativa, la cual les produce y aumenta la desmotivación. 
Entre las causas del problema se argumenta, la falta de estrategias de adecuadas para una clase, las cuales se emplean para el desarrollo del pensamiento creativo; a fin de enfrentar dignamente las exigencias requeridas en la transversalidad del proceso de enseñanza - aprendizaje. Por tales razones, el profesor amerita disponer de información concreta que ayude al alumno de media general a pensar, comprender e imaginar el significado de lo aprendido.

Las políticas de las organizaciones educativas, centradas en los aspectos materiales y no en el material humano (profesores-alumnos), la falta de actualización de los profesores en metodologías activas de enseñanza, la improvisación en la selección de los temas seleccionados, muchos docentes tienen debilidad en la formación pedagógica, en el conocimiento base, la continuidad de patrones de enseñanza; constituyen las posibles causas de tal atraso, una realidad existente en el Ministerio del Poder Popular para la Educación en general.

En el municipio San Francisco, la praxis educativa del profesor tiene debilidades en la reflexión sistemática. Esto se evidencia en la falta de fundamentación teórico - práctico en la toma de decisiones y en el conocimiento por aprender, además, este problema no es exclusivo de la profesión docente, es transversal al desarrollo profesional en diversos contextos.

En relación con lo anterior expuesto, a través de visitas no programadas y entrevistas no estructuradas a los liceos del Municipio N ${ }^{a}$ 1, de San Francisco de los liceos de media general los docentes de ciencias sociales, manifiestan una disminución en el ritmo de trabajo, falta de interés en la realización de las tareas asignadas, sensación de desagrado por la calidad de la educación que afecta la formación integral del docente. Asimismo, en la aplicación del currículo general dentro de cada asignatura se observa una relación aislada de los contenidos de enseñanza dentro de las unidades didácticas, carente de sentido, la falta de formación técnica y profesional, resistencia al cambio de mentalidad conservadora, para una mentalidad creativa. 
Estrategias de Enseñanza y Creatividad del Docente en el área de Ciencias Sociales de Instituciones Educativas de Media de San Francisco 1

Otros factores que influyen en la conducta poco creativa del docente, es la vocación, la mayoría de los profesores que están egresando de las universidades y misiones no se están formando para la enseñanza, sino para devengar un sueldo que les sirva para vivir, a esto se le suma, la inconformidad de ser el gremio peor pagado, todavía no existe una explicación para ello, pero si no hay docentes motivados y creativos, faltaran en las aulas alumnos innovadores y constructores de sus propias ideas.

Ante esta situación, los factores descritos posiblemente generen en el docente un cambio en la concepción del proceso de enseñanza - aprendizaje, de la transición del saber a la construcción del conocimiento, y el papel del profesor sólo se centra en trasmitir conocimientos, sin preocuparse por el aprendizaje significativo del alumno. En consecuencia, lo descrito posiblemente genere falta de motivación, interés, apatía tanto en el estudiante como en el docente.

Por tal razón, se hace necesario determinar la relación entre las Estrategias de Enseñanza y la Creatividad del Docente en el área de Ciencias Sociales de las Instituciones Educativas de Media General del Municipio San Francisco 1, estado Zulia, cuya aplicación en el contexto educativo debe ser adecuada, permitiendo innovar en la práctica pedagógica para beneficio de los actores del proceso de enseñanza - aprendizaje. Aunado a esto, la praxis del profesor va a tener relevancia, si se utiliza la estrategia adecuada en el aula de clase.

Aunado a la idea anterior, se deben formular lineamientos prácticos para la optimización de las estrategias de enseñanza y creatividad del docente en área de ciencias sociales de instituciones educativas de media general del municipio San Francisco 1, estado Zulia. Es por ello que se requiere un profesor mediador, que desarrolle habilidades en los discentes para acceder al conocimiento eficazmente. Este mediador, facilitador y orientador debe ser creativo para implementar diferentes estrategias en el aula e innovar en sus procesos de enseñanza continuamente, según lo requieran los estudiantes. 


\section{Estrategias de enseñanza.}

El docente como facilitador del aprendizaje debe conocer los intereses de los discentes (inteligencia múltiple), y los diversos estímulos de sus contextos: Familiares, comunitarios, educativos entre otros, además de realizar las actividades para que los estudiantes investiguen, descubra y compartas sus ideas. En opinión de Gouveia y Atencio (2012), sostienen:

"Las estrategias de enseñanza contempla dos dimensiones: una que se caracteriza por su naturaleza prescriptiva, es decir, son aquellos procedimientos, modelos o formas de proceder determinados de antemano para realizar la enseñanza. La segunda dimensión está caracterizada como un proceso constructivo, en ella se recuperan los procesos espontáneos, constructivos y cotidianos" (p. 20)

De esta forma, se puede afirmar cuando los docentes explican una estrategia para la enseñanza, primero identifican qué van a enseñar y luego eligen la relacionan con el objetivo. Está diseñada específicamente para lograr un objetivo particular y determinará gran parte de las acciones del profesor, convirtiéndose en una especie de proyecto.

De esto se señala, el carácter flexible de la estrategia, característica que recupera el determinismo, enfrentándolo con el momento, reelaborándolo de acuerdo a la necesidad de la situación de enseñanza. Asimismo, éstas están consideradas como la organización metódica de las secuencias de aprendizajes; siendo procesos de intercambio y comunicación, los recursos didácticos, el sistema de evaluación, en función del ambiente de aprendizaje y de los objetivos propuestos. Cada estrategia de enseñanza supone una concepción de proceso educativo y de una explicación.

En este sentido, de acuerdo con Díaz y Hernández (2010, p. 58), “son procedimientos utilizados por el agente de enseñanza en forma reflexiva y flexible para promover el logro de aprendizajes significativos en los alumnos". Además, las estrategias de enseñanza, según Arévalo (2008, p.119), constituyen "un conjunto de medios operativos usados para el logro de procedimientos que deben seguirse para recorrer las diferentes etapas del proceso enseñanza-aprendizaje". Mientras, Bencomo (2008, p.99), son "el conjunto de medios y 
Estrategias de Enseñanza y Creatividad del Docente en el área de Ciencias Sociales de Instituciones Educativas de Media de San Francisco 1

procedimientos que se utilizan para el logro de la enseñanza-aprendizaje de manera didáctica, incluyendo la tecnología educativa".

En este orden, es necesario establecer la praxis de enseñanza más efectiva para desarrollar estrategias de comprensión de textos escritos en alumnos del segundo ciclo de educación media general, los profesores plantearon problemas que requerían de la aplicación de las lecturas discutidas en la clase. En segundo lugar, se enfatizaba el aprendizaje cooperativo y la enseñanza recíproca.

Del mismo modo, Cormack (2009), afirma la acción educativa también implica al docente y la enseñanza aunque de manera distinta a la tradicional. Para este autor, las estrategias de enseñanza son "como un conjunto de ayudas que el docente brinda al discente en la realización de las actividades, en su proceso personal de construcción de conocimientos". Estas estrategias de enseñanza deben ser consignadas en la programación como una parte importante de la misma, porque es un elemento nuevo incorporado y es necesario seleccionarlo previamente, para saber cuál utilizará en cada caso, de tal manera que su acción sea dinámica.

Según Montes y Machado (2011), las acciones de los alumnos dadas durante el aprendizaje e influyen en la motivación, la asimilación, la interpretación, la retención o la transferencia de la información, toda estrategia de enseñanza y de aprendizaje se encuentran involucradas, entre enseñar y aprender. Sin embargo, por lo que cada vez es más frecuente el uso de la expresión estrategias de enseñanzaaprendizaje, las cuales pueden ser consideradas como secuencias integradas, extensas o complejas, de acciones, procedimientos seleccionados y organizados, atendiendo a todos los componentes del proceso, las mismas persiguen alcanzar los fines de la educación propuestos.

Sobre la base de lo antes expresado, las estrategias de enseñanza, como un conjunto de procedimientos, son respuestas al cómo hacer, para alcanzar un fin o resultado propuesto, orientándose al logro de cada etapa del proceso enseñanzaaprendizaje, complementada con la tecnología educativa, con una praxis pedagógica reflexiva y consciente. Aunado a esta idea, el profesor debe estar contantemente 
motivado para hacer las clases dinámicas para que sus discentes sean constructores de su propio aprendizaje, socializando e innovando.

\section{EstrategiasPreinstruccionales}

Las estrategias de enseñanza preinstuccionales, son las usadas por el docente con la intención de facilitar el aprendizaje significativo de los estudiantes. Estas han demostrado, en diversas investigaciones su efectividad al ser introducías como apoyo en texto académico así como en la dinámica de la enseñanza.

Al respecto, Díaz y Hernández (2010, p. 143), refieren las estrategias preinstruccionales son "una preparación o alerta al estudiante en cuanto a los conocimientos o experiencias previas que le van a permitir ubicarse en el contexto del aprendizaje asertivamente como los objetivos y el organizador previo". Aunado a esto, el docente debe realizar la diagnosis correspondiente antes de iniciar su clase correspondiente.Del mismo modo, los autores Bustamante, Carmona y Renteria (2011), afirma que estrategias preinstruccionales hacen énfasis a la manipulación de objetos, textura, color y forma. En primera instancia, cuestionan e inquietan al estudiante frente a la temática a desarrollarse y la forma en como se aprenderá, su tarea principal es activar el conocimiento que se posee frente a una temática y a su vez enfrentar al estudiante con sus capacidades cognitivas e intelectuales.

Para que esta se implemente adecuadamente, se debe desarrollar una buena estructuración partiendo de unos objetivos, los cuales mostrarán el camino al cual se quiere llegar, además dan a conocer la finalidad del material y cómo manejarlo, y activan los organizadores previos, que se convertirán en abrebocas del tema, el cual motiva al estudiante a querer saber mucho más del contenido y de esta manera entrar a realizar un buen desarrollo de clase.

En este grupo, se incluyen a aquellas otras que se concentran en el esclarecimiento de las intenciones educativas que el profesor pretende lograr al término de la situación educativa. Se recomienda usarlas sobre todo al inicio de la clase. Ejemplos de ellas son: las interrogantes, la actividad generadora de información 
Estrategias de Enseñanza y Creatividad del Docente en el área de Ciencias Sociales de Instituciones Educativas de Media de San Francisco 1

previa. (Lluvia de ideas, los enunciados de objetivos, entre otros). En este sentido, estas estrategias como aquellas que regularmente preparan y alertan al alumno en cuanto a con qué y cómo va a aprender; en tal sentido, tratan de incidir en la activación o la generación de los conocimientos conjuntamente con experiencias previas necesarias, ubicando al estudiante en el contexto conceptual apropiado, generando así, expectativas adecuadas facilitando el aprendizaje significativo de los mismos.

\section{Estrategias Coinstruccionales}

De igual manera las estrategias coinstruccionales orientan la atención de los estudiantes, tales estrategias son aquellos recursos que el profesor o el facilitador utiliza para mantener la atención de los alumnos durante un discurso. En cuanto a las estrategias construccionales, Díaz y Hernández (2010, p.143), afirman "son aquellas que apoyan los contenidos curriculares durante el proceso de enseñanza o de la lectura del texto que se enseña".

Además, tienen instrumentos tales como: mapas conceptuales y analogías, ilustraciones, entre otras. En tal sentido, los autores señalan que entre las funciones de estas estrategias están: la de detección de la información principal; conceptualización de contenidos; delimitación de la organización, estructura e interrelaciones entre dichos contenidos y mantenimiento de la atención y motivación.

De tal forma, Bustamante, Carmona y Renteria (2011), usan las estrategias para mejoramiento de la recepción de contenidos, captando la idea central, la cual le permita cuestionarse hasta llegar a relacionar la de mayor importancia para desarrollar las competencias interpretativa, prepositiva y argumentativa, logrando la comprensión progresiva del conocimiento a través de diversas actividades que interioricen, cuestionen y motiven al sujeto.

En estas se incluyen los mapas conceptuales, reflejando un esquema de conocimiento obtenido por medio de ilustraciones, las cuales facilitan la codificación 
visual de la información. Además, son representaciones visuales de situaciones reales y concretas, que enfrentan al sujeto con la realidad. También, las analogías desarrollan el pensamiento lógico semejante una cosa a otra, con estas estrategias se identifican aquellos pensamientos mentales desarrollados por el sujeto, para tener un aprendizaje significativo y a la vez efectivo, activando sus habilidades.

Por lo tanto, las estrategias coinstruccionales son vistas como aquel recurso que el docente usa para mantener la atención de los estudiantes durante una clase. En este sentido, pueden aplicarse de manera continua para indicar a los alumnos sobre los conceptos o ideas y deben centrar sus procesos de atención, codificación, aprendizaje. Algunas estrategias que pueden incluirse son las siguientes: los gráficos, redes semánticas e ilustraciones.

En el contexto actual, las instituciones educativas de media general, no cuentan con personal motivado para generar nuevos conocimientos en los alumnos, dificultando el desarrollo del pensamiento creativo de los mismos, porque los docentes se preocupan por explicar contenidos sin realizar análisis e interpretaciones de los temas planificados.

\section{Estrategias Posinstruccionales}

Refiriéndose a las estrategias posinstruccionales, Díaz y Hernández (2010, p.143), "las presentan después de los contenidos que se han de aprender, y que a su vez permiten al alumno formarse una visión sintética, integradora e incluso critica del material". Entre algunas de las mencionadas estrategias están: resúmenes finales, redes semánticas y mapas conceptuales.

Del mismo modo los autores Bustamante, Carmona y Renteria (2011), expresan que estas le permiten al estudiante sea autónomo en su proceso, debido a que este momento, tiene una visión integradora, confrontando sus propios conocimientos, para darle valor a lo que se sabe, realizando sus propias hipótesis y apoye o refute diversas ideas. 
Estrategias de Enseñanza y Creatividad del Docente en el área de Ciencias Sociales de Instituciones Educativas de Media de San Francisco 1

Entre las estrategias posinstruccionales más conocidas, se encuentran: los resúmenes, en ellos lo que prevalece es la síntesis y la información relevante de un texto, teniendo en cuenta ideas primarias, secundarias, premisas y conclusiones. Los organizadores gráficos: donde se realizan representaciones visuales de palabras y explicaciones de un texto.

Según Villalobos (2010, p. 2), estas "se presentan después del contenido que se ha de aprender y permiten al alumno formar una visión sintética, integradora e incluso critica del material". En otros casos le permiten valorar su propio aprendizaje. Algunas de las estrategias posinstruccionales más reconocidas son resúmenes, redes semánticas y mapas conceptuales.

Desde este contexto, este tipo de estrategias posinstruccionales, se presentan al terminar el episodio de enseñanza, permitiendo al discente, en algunos casos, apreciar su propio aprendizaje, siendo estos significativos para ellos y el profesor que imparte la cátedra diariamente en las instituciones educativas. Por tal razón, los docentes de algunas instituciones no hacen el cierre para saber y constatar si se ha retroalimentado al alumno, donde éste debe haber codificado los mensajes o explicaciones del profesor, mediante la comprensión, estrategia de enseñanza utilizada, recursos y técnicas, que son los elementos fundamentales en el aprendizaje.

\section{Creatividad del Docente}

Para Correa (2010, p. 2), la creatividad es definida como "la facultad de crear en general, o la capacidad para realizar obras artísticas u otras cosas por medio de la imaginación". En tal sentido, la creatividad es un fenómeno en el que intervienen elementos características de lo creativo como: espontaneidad, sentido del humor, curiosidad intelectual, iniciativa, intuición, independencia, inteligencia, persistencia, constancia y constituye un aspecto importante de la solución de problemas.

A su vez, las individuos creativos son aquellos que dan lugar con frecuencia a productos creativos, es decir, originales, caracterizados por una combinación de inteligencia y personalidad en la que influyen las relaciones con otros profesionales, las 
circunstancias de trabajo y el apoyo de los que rodean al individuo creador e influyen significativamente en el proceso creativo.

En tal sentido, De Bono (2009, p. 85), relaciona a la creatividad con "el pensamiento lateral siendo necesario ampliar la visión, enriqueciendo el uso de la información antes de pensar de manera vertical". La base de su método para desarrollar la creatividad consiste en introducir pautas como elementos activadores del pensamiento, produciendo un cambio de "penetración" y que proporcionan alternativas distintas y originales.

De igual manera el autor, sugiere el uso de herramientas del pensamiento, permitiendo, sacar a las personas de los extremos para explorar nuevos caminos, utilizar la información de otra manera y reestructurar sus patrones de los mismos. Igualmente, considera que la percepción es muy importante en las primeras etapas de todo proceso de pensamiento, favoreciendo la posibilidad de generar ideas que aun cuando parezcan ilógicas contribuyen a desarrollar la creatividad.

Al respecto, López (2009, p. 58), la creatividad es "la capacidad de crear, de producir cosas nuevas, para llegar a conclusiones y resolver problemas en una forma original”. Así, la misma es el principio básico para el mejoramiento de la inteligencia personal y del progreso de la sociedad, y es también, una de las estrategias fundamentales de la evolución natural.

Por último, el concepto de creatividad es diferente para diversos contextos donde, la actividad humana expresada en el concepto puede ser muy variada. En cualquier caso, pueden establecer límites para el empleo de esta palabra, aunque las delimitaciones son confusas, indeterminadas, relacionada con la innovación para generación de conceptos nuevos de diversos temas.

Ante lo expuesto, los docentes no desarrollan la creatividad de sus alumnos ni la de ellos, lo cual ha generado un sinnúmero de obstáculos en el proceso de enseñanzaaprendizaje, dificultando la retroalimentación, pensamiento creativo, producción de ideas innovadoras, fluidez, originalidad, sensibilidad, flexibilidad y por último, la iniciativa que deben tener en el entorno escolar. 
Estrategias de Enseñanza y Creatividad del Docente en el área de Ciencias Sociales de Instituciones Educativas de Media de San Francisco 1

\section{Facetas de la Creatividad}

De igual manera, para Díaz (2008, p. 45), una faceta es un aspecto que puede considerarse de un asunto, una persona. Por lo tanto, puede ser un aspecto o punto de vista de una cuestión. Esto puede considerarse desde una perspectiva o teniendo en cuenta diversos criterios Las diferentes características de los seres humanos, pueden mencionarse como facetas. Los individuos son unidimensionales, más allá de las especializaciones, todos pueden desarrollar diversas actividades

Seguidamente, Montero (2006, p. 3), refiere que "la faceta está compuesta por un conjunto de categorías. Según como sean las relaciones entre las categorías que formen las facetas, pudiendo diferenciar básicamente entre: (a) Faceta Jerárquica: Las categorías poseen relaciones de tipo jerárquico (parte-todo, general-específico); (b) Faceta Plana: Al margen de la relación (implícita) que les confiere pertenecer a una misma faceta, las categorías no tienen ningún tipo de relación (explícita) entre ellas. (c) Otras: Las categorías poseen otro tipo de relaciones (explícitas) a parte de las de tipo jerárquico (por ejemplo, relaciones de tipo asociativo).

Las mismas son contenedores mutuamente excluyentes de categorías. Esto quiere decir, una misma categoría no puede pertenecer a dos facetas diferentes, entonces, puede perder su función: caracterizar un objeto desde diferentes puntos de vista, para así, obtener una descripción global y multidimensional del objeto. Por tal razón, cuando se es creativo, el pensamiento aborda la realidad concreta que los envuelve, para así innovar en el salón de clase según el tema explicado.

Según Blanquez (2009, p. 3), la creatividad es "el ingrediente esencial de toda propuesta interior que requiere desarrollarse, practicarse y estar expuesto ante diferentes tipos de estímulos a la ruptura de esquemas y de la organización no común de lo ya conocido para que pueda sensibilizar su desarrollo". Para ser creativos, es necesario llegar a conocer las fases de este proceso, su funcionamiento, aunque no es un requisito necesariamente productivo para que los discentes consigan este objetivo, porque los logros creativos y los grandes genios existen desde hace años, sin que ellos tuvieran conciencia de las fases de este proceso. 
Para finalizar, las facetas de la creatividad consisten en diversos estadios o etapas por donde pasa el proceso creativo desarrollado por los individuos en este caso docentes y alumnos en las instituciones educativas, permitiendo asociar, innovar, contrastar, analizar temas, informaciones o conocimientos expresados en los contextos organizacionales. En el mismo orden de ideas, los docentes deben desarrollar su pensamiento creativo, considerando las necesidades actuales de los alumnos y de ellos mismos, las cuales están sujetas a la realidad existente en el contexto escolar, asimismo, los directivos deben fomentar la participación y la innovación de ideas, actividades, proyectos, en concordancia con la comunidad, para lograr espacios armónicos en el proceso de enseñanza-aprendizaje.

\section{Indicadores de la Creatividad}

De acuerdo a lo ya mencionando, la palabra creatividad surge como una necesidad esencial, exigida al discente al momento de dar la clase, estableciendo los tipos de procesos que puedan facilitar el desarrollo de la misma, determinando las habilidades básicas que los alumnos deben poseer, resulta una tarea muchas veces postergada o relegada en relación a indicadores concretos. Para Corbalán (2003, p.18), los indicadores son "grados o niveles de creatividad que existen para describir a las personas".

Con este planteamiento, se refiere a las condiciones de todas las personas. De esta forma, se afirma, lo existente son diferentes grados de creatividad en los individuos. También Penagos y Aluni (2005, p 18), expresan todas las personas son creativas, aunque sí es posible establecer niveles de creatividad y reconocer a individuos, excepcionalmente dotados que crean cosas, ideas, que en algunos de los casos, el docente queda maravillado ante tanta imaginación.

En el mismo orden de ideas, los indicadores de la creatividad son diversos estadios o factores que poseen las personas creadoras de ideas y pueden plasmarse mediante una valoración y comprensión del proceso creador. Igualmente, ayudan a reconocer a las personas brillantes, interesantes y estimuladoras en el contexto social. 
Estrategias de Enseñanza y Creatividad del Docente en el área de Ciencias Sociales de Instituciones Educativas de Media de San Francisco 1

A tal efecto, en las organizaciones educativas, los docentes no miden los indicadores de la creatividad en ningún aspecto, porque estos no se interesan si los alumnos son creativos, o si en alguna de las clases innovan conocimientos sobre los temas, solamente los explican de manera tradicional, evaluando conceptos mediante una o dos pruebas, además, de utilizar simplemente una misma técnica o recurso.

\section{Metodología}

El tipo de estudio se ubicó entre el descriptivo - correlacional, ya que el estudio en sus objetivos se centró en precisar como es y se comportan las variables en el contexto de estudio. Es decir, se caracterizaron y describieron situaciones que develaron tantas estrategias de enseñanza, así como creatividad del personal que ocupa las funciones de docente de ciencias sociales en educación media general. Asimismo, se consideró correlacional por lo que este tipo de estudio tiene como finalidad determinar el grado de relación o asociación no causal existente entre dos o más variables.

A su vez, el diseño fue no experimental, porque sólo se observaron los fenómenos tal como se presentan en su contexto natural, sin manipulación deliberada de las variables. De igual modo, se consideró de campo, pues se efectuó en el mismo contexto donde las variables de estudio se expresan. La población estuvo conformada por tres (3) directivos, noventa y tres (93 docentes) y trescientos (300), estudiantes en los diferentes líceos Eduardo Mathías Lossada, Evaristo Fernández y José Antonio Calcaño. Estos líceos fueron seleccionados al azar. Además, se consideró el censo poblacional por ser necesario todos los elementos de la población, cuya muestra fue intencional u opinática, finita y accesible.

Se utilizó como técnica de observación por encuestas, para definir uno de los indicadores y facilitar la medición de las variables. De igual manera, se diseñó un cuestionario el cual estuvo dirigido al personal directivo y docente en el área de ciencias sociales, constituido por 45 items basados en un tipo de escala de frecuencia con alternativas de respuestas: siempre, casi siempre, algunas veces, casi nunca y 
nunca, con la siguiente ponderación que van de 1 hasta 5. Igualmente, se elaboró otro para los estudiantes con las mismas características nombradas anteriormente.

\section{Resultados}

En relación a las estrategias preinstruccionales, que la mayoría de los docentes $(67,8 \%)$ considera que nunca se utilizan estrategias como el diseño de objetivos, organizadores previos o señalizaciones durante el proceso de educativo, además, el $22,9 \%$ de los docentes opina que casi nunca se emplean estas estrategias, sólo el 8,2\% lo hace algunas veces y casi siempre el 1,1\%.

Por otro lado, el $42,7 \%$ de los estudiantes encuestados consideran que algunas veces los docentes desarrollan las estrategias como se indica en el párrafo anterior. Pero, el 37,8\% señala que casi nunca lo hacen y el $4 \%$ nunca. También, otro $8 \%$ señaló que casi siempre se cumple con lo planteado y siempre según el 7,6\%.

Estos resultados demuestran que en estas instituciones educativas el personal aún no ha comprendido la importancia del uso de estrategias preinstruccionales, a fin de facilitar el aprendizaje significativo de los estudiantes, tal como lo indica Díaz y Hernández (2010, p. 143), cuando expresa que estas estrategias provocan "una preparación o alerta al estudiante en cuanto a los conocimientos o experiencias previas que le van a permitir ubicarse en el contexto del aprendizaje asertivamente", lo cual podría provocar apatía en el estudiantado, poco interés por aprender y predisposición negativa frente a las actividades a desarrollar en clase.

Con respecto a las estrategias posinstruccionales, evidencia el $54,1 \%$ de los docentes asegura que algunas veces se utilizan los mapas conceptuales, resúmenes o analogías como herramienta de aprendizaje y promueve su uso como método para ayudar a los estudiantes en captar el significado de los materiales que se van aprender. Sin embargo, el $28,2 \%$ junto al $15,5 \%$ de los docentes casi nunca y nunca los utiliza. 
Estrategias de Enseñanza y Creatividad del Docente en el área de Ciencias Sociales de Instituciones Educativas de Media de San Francisco 1

Pero, el 58,2\% de los estudiantes indicó que algunas veces los docentes prefieren estas estrategias, el $22,7 \%$ y el $9,8 \%$ indicaron que casi nunca y nunca las utilizan, aunque el $7,1 \%$ y el $2,2 \%$ restante siempre y casi siempre trabajan con estrategias posinstruccionales.

El uso de las estrategias de enseñanza fueron catalogadas por los docentes como Muy Deficiente, mientras que los estudiantes consideran que su uso es aceptable, lo cual predice serios inconvenientes, atendiendo a lo planteado por Cormack (2009) ya que desde una perspectiva pedagógica el maestro debe ofrecer al alumno el apoyo necesario para restablecer el equilibro, y las estrategias de enseñanza son "como un conjunto de ayudas que el docente brinda al discente en la realización de las actividades, en su proceso personal de construcción de conocimientos.”.

En relación a la variable Creatividad del docente, entre las facetas de la creatividad, el $56,87 \%$ de los docentes considera que algunas veces estimulan la creatividad del estudiante mediante el ingenio, la flexibilidad y la genialidad. Sin embargo, de acuerdo con el $22 \%$ y el $11 \%$ casi nunca y nunca se aprecian las facetas, sólo lo hacen el 4,2\% y un 1,8\% casi siempre y siempre. Además, el 62,7\% de los estudiantes opina igualmente que algunas veces los docentes promueven el ingenio, la flexibilidad y la genialidad en la institución. Le sigue el $20 \%$ que respondió casi nunca, el 7,1\% casi siempre, el 5,3\% nunca y el 4,9\% siempre.

En relación a los indicadores de la creatividad, el 60,8\% de los docentes considera que algunas veces se observa originalidad, comunicación e innovación en el alumno, pero el 18,97\% casi nunca lo aprecia y nunca el 10,2\%. Sin embargo, el 5\% casi siempre percibe originalidad y el $4 \%$ siempre lo hace.

Por otro lado, el 55,6\% de los estudiantes opina que algunas se emplea la originalidad, la comunicación y la innovación, pero el 25,3\% dice que casi nunca y el $7,6 \%$ nunca. Además, el 7,1\% de los estudiantes encuestados consideran que siempre se observan estos indicadores y casi siempre según el 4,4\%. 
Finalmente, la Variable Creatividad del Docente fue catalogada por ambos grupos como aceptable, mostrando similitud con los hallazgos obtenidos por Morón (2009) cuyas conclusiones indicaron que los docentes y estudiantes coincidieron en que los tipos de estrategias predominantes son las expositivas, estructurales y participativas, mientras que, las creativas son las menos incluidas en la instrucción.

Por último, a fin de determinar la relación entre las estrategias de enseñanza y la creatividad de los docentes en los liceos seleccionados, se calculó la fórmula de Pearson, la cual arrojó un valor de 0,93. Estos valores indican que existe una relación positiva y fuerte entre estas variables. Es decir, que a medida que aumenta el uso por parte de los docentes de estrategias de enseñanza, en esa misma medida aumenta su creatividad y viceversa, si la primera disminuye la segunda disminuirá.

Todo lo anterior confirma la premisa de la investigación, ya que la creatividad de los docentes depende del conocimiento e importancia que le den al uso de las estrategias, si descuidan su debida planificación, no será posible alcanzar altos niveles de creatividad en el estudiantado.

\section{Conclusiones}

Una vez analizados y discutidos los resultados de la investigación se llegó a las siguientes conclusiones:

Al identificar las Estrategias Preinstruccionales utilizadas por el Docente en el área de Ciencias Sociales, se concluye que muy poco se utilizan los objetivos, los organizadores previos y las señalizaciones en las Instituciones Educativas de Media General del Municipio San Francisco 1.Estado Zulia.

Con respecto a las Estrategias Coinstruccionales utilizadas por el Docente en el área de Ciencias Sociales, se encontró que las más utilizadas fueron las redes semánticas y las ilustraciones de acuerdo a la opinión de los estudiantes, pero los 
Estrategias de Enseñanza y Creatividad del Docente en el área de Ciencias Sociales de Instituciones Educativas de Media de San Francisco 1

docentes aseguran que poco se utilizan estas estrategias en las Instituciones Educativas de Media General del Municipio San Francisco 1. Estado Zulia.

En cuanto a las Estrategias Posinstruccionales utilizadas por el Docente en el área de Ciencias Sociales, se concluye que se emplean medianamente los mapas conceptuales, los resúmenes y las analogías, en Instituciones Educativas de Media General del Municipio San Francisco 1. Estado Zulia. Siendo las estrategias posinstruccionales las mayormente empleadas.

Se concluye que para los estudiantes las estrategias empleadas en la escuela son aceptables, mientras que para los docentes dichas estrategias son muy deficientes, estas divergencias indican una sentida necesidad de mejoramiento de las estrategias empleadas por parte del personal docente.

$\mathrm{Al}$ analizar las Facetas de la Creatividad del Docente en el área de Ciencias Sociales, se concluye que los docentes medianamente demuestran y promueven ingenio, flexibilidad y genialidad en las Instituciones Educativas de Media General del Municipio San Francisco 1. Estado Zulia.

Con respecto a los Indicadores de la Creatividad del Docente en el área de Ciencias Sociales, se tiene que la misma fue catalogada como Mediana, ya que los valores de la originalidad, la comunicación y la innovación fueron intermedios en las Instituciones Educativas de Media General del Municipio San Francisco 1. Estado Zulia.

Finalmente, al establecer la relación entre Estrategias de Enseñanza y Creatividad del Docente en el área de Ciencias Sociales de Instituciones Educativas de Media General del Municipio San Francisco 1. Estado Zulia, se concluye que existe una relación positiva y fuerte entre estas variables, es decir, que a medida que aumenta el uso por parte de los docentes de estrategias de enseñanza, en esa misma medida aumentará su creatividad y viceversa, si la primera disminuye la segunda disminuirá. 


\section{Referencias Bibliográficas}

Arévalo, Marcos. (2008). Estrategias Didácticas: Una visión Alternativa e Innovadora. Editorial Magisterio. Colombia.

Bencomo, Luis. (2008). Métodos, Técnicas y Estrategias. Editorial Magisterio. Colombia.

Blanquez, Diego. (2009). Tecnología e Innovación. Profit. España.

Bustamante, Paula., Carmona, Maritza. y Reinteria, Yuli. (2011). Importancia de las estrategias de Aprendizaje en el Desarrollo de los Procesos de Enseñanza. Fundación Universitaria Luis Amigo. Medellín.

Cerda, Hugo (2005). La Creatividad en la Ciencia y en la Educación. Aula Abierta. Magisterio. Colombia

Cormack, Lynch. (2009). Estrategias de Aprendizaje y de Enseñanza en la Educación menor de 6 años. Acción Pedagógica. Vol. 3 № 6. Venezuela (Pp. 267-283).

Correa, Luis. (2010). Creatividad y Cerebro. Profit. España.

De Bono, Edward. (2009). Creatividad. 62 Ejercicios para Desarrollar la Mente. McQuaigGroup, Inc. España

Díaz, Frida, y Hernández, Geraldo. (2010). Estrategias docentes para un aprendizaje significativo. Mc Graw Hill Interamericana. México.

Díaz, Margarita. (2008). Creatividad y Educación. Fareso. España.

Goveia, Edth. y Atencio, Maxula (2012). Estrategias Didácticas para las Ciencias Sociales. Ediluz. Venezuela

López, Salvador. (2009). Estética de la Creatividad. Arte Literatura. Mc Graw Hill. México.

Montero, Laura. (2006). Creatividad ¿Cómo Desarrollarla? Editorial Pax. México. Montes, Susana. y Machado, Raynier. (2011). Estrategias en Educación. Profit. España.

Morón, Pablo. (2009). Creatividad del docente y desempeño laboral en instituciones de educación inicial. UNERB: Venezuela

Penagos, Martha. y Aluni, Raúl.,(2000). Preguntas más frecuentes sobre creatividad. Revista Psicología, (ed. Especial). (Pp.113-130).

República Bolivariana de Venezuela (2007-2013) Proyecto Nacional Simón Bolívar Primer Plan Socialista. Proyecto Simón Bolívar. Presidencia de la República. Venezuela.

Villalobos, Gerardo. (2010). Una Interpretación Constructivista de la enseñanza. Pax. México. 